\title{
High Energy Electron Radiation Exposure Facility at PSI
}

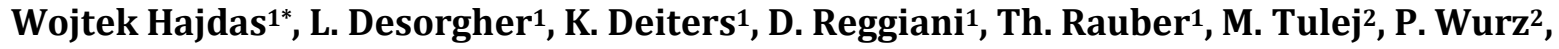 \\ M. Luethi' ${ }^{2}$ K. Wojczuk' ${ }^{1}$ P. Kalaczynski ${ }^{1}$ \\ ${ }^{1}$ Paul Scherrer Institut (PSI), Villigen, Switzerland \\ ${ }^{2}$ University of Bern, Bern, Switzerland \\ Email: wojtek.hajdas@psi.ch
}

Received 3 June 2014

\begin{abstract}
Paul Scherrer Institut hosts the Proton Irradiation Facility used for radiation effects studies and exposure tests in preparation of satellite missions for the European Space Agency. The facility allows for realistic simulation of the space proton spectra in the energy range from $6 \mathrm{MeV}$ up to 230 $\mathrm{MeV}$ with exposure fluxes ranging from very low up to as high as $10^{9} \mathrm{p} / \mathrm{cm}^{2} / \mathrm{sec}$. Recently, approved ESA mission to Jupiter-JUICE-also brought a need for tests with high energy electron beams. For this purpose, another facility was established in the PSI secondary beam area piM1. Secondary particles are produced on the thick carbon target hit by energetic proton beam. Dedicated beam optics enables selection of the particle charge and momentum and guides them to the tests area. Characterization of electron beams at various momenta was performed with respect to their intensity, profiles and contamination by pions and muons. Electron fluxes ranging from $1.5 \times$ $10^{3} / \mathrm{cm}^{2} / \mathrm{s}$ at $20 \mathrm{MeV} / \mathrm{c}$ to $2.3 \times 10^{6} / \mathrm{cm}^{2} / \mathrm{s}$ at $345 \mathrm{MeV} / \mathrm{c}$ with gaussian beam profiles with FWHM of about $4 \mathrm{~cm}$ were measured. Beam contamination with heavier particles becomes negligible for all momenta lower than $115 \mathrm{MeV} / \mathrm{c}$. This allows for using them for components and shielding characterization and detector calibration experiments. Several such experiments have been already performed utilizing available beam time of few weeks per year.
\end{abstract}

\section{Keywords}

Irradiations, Electron Beams, Components Tests, Exposure Facilities

\section{Introduction}

Secondary beam experimental areas of PSI are mainly used for basic research tests with pions and muons. They utilize $590 \mathrm{MeV}$, high intensity proton beam from the synchro-cyclotron and two carbon targets used to produce secondary particles. Several beam lines were constructed and specialized in delivering of various secondary particles with respect to the type, charge and momentum. Design and complexity of each beam line influence such beam parameters as particle intensity, range of its momenta and momentum resolution as well as contamination

${ }^{*}$ Corresponding author.

How to cite this paper: Hajdas, W., Desorgher, L., Deiters, K., Reggiani, D., Rauber, Th., Tulej, M., Wurz, P., Luethi, M., Wojczuk, K. and Kalaczynski, P. (2014) High Energy Electron Radiation Exposure Facility at PSI. Journal of Applied Mathematics and Physics, 2, 910-917. http://dx.doi.org/10.4236/jamp.2014.29103 
degree with other particle types. Although most of the experiments are devoted for studies of classic particle physics problems, some of the recent activities also cover detector and shielding characterization tests for space missions. An example of such mission is JUICE planned to be sent by the European Space Agency to Jupiter for studies of its icy moons and search for life-friendly inhabitants. Radiation environment of Jupiter is especially severe and not well characterized yet. Values of absorbed total dose expected within the JUICE mission lifetime are bigger than $1 \mathrm{Mrad}(\mathrm{Si})$ while anticipated energies of trapped electrons are even higher than one hundred $\mathrm{MeV}$. It poses a great challenge to all subsystems and components of the spacecraft even if they are categorized as radiation resistant for standard missions around earth. Therefore, qualification of them for JUICE requires dedicated and extended studies. In particular, instruments concept, operating conditions, quality of shielding and performance of any detection system onboard, also including radiation monitors, must go through careful verification and optimization. It also requires new exposure facilities available for at least several weeks per year and able to deliver electron beams with energies and intensities enabling for instrument tests in mission semi-realistic or anticipated conditions. The piM1 secondary experimental area of PSI was selected for this purpose. Its beams were precisely characterized and optimized for future experiments dedicated to space research. All steps leading to the facility characterization and commissioning are described in the following chapters.

In the first step, the existing infrastructure is outlined and all optimization measures for detection of electrons are detailed in chapter 2 . Several measurements using dedicated experimental techniques and including optimized arrangement for user test systems are described in chapter 3 . They include studies of electron beam and separation of other particles. Experimental results and characteristics of the available test beams are provided in chapter 4 . The last chapter 5 contains concise summary and future plans.

\section{Secondary Beam Area piM1}

Secondary particles for the Large Research Facilities at PSI are created by protons passing through $2 \mathrm{~mm}$ thick graphite target located in the main beam-line of the PSI synchro-cyclotron. High energy and intensity of the primary proton beam, $590 \mathrm{MeV}$ and $2 \mathrm{~mA}$ respectively, allows for very efficient production of mainly pions, muons but also electrons. The beam of secondary particles enters the path to the piM1 area at the angle of 22.3 degree with respect to the initial proton beam. The piM1 area layout is shown in Figure 1 [1]. Its selection for space experiments was based on the spectral characteristics of its pion and muon beams with their intensity

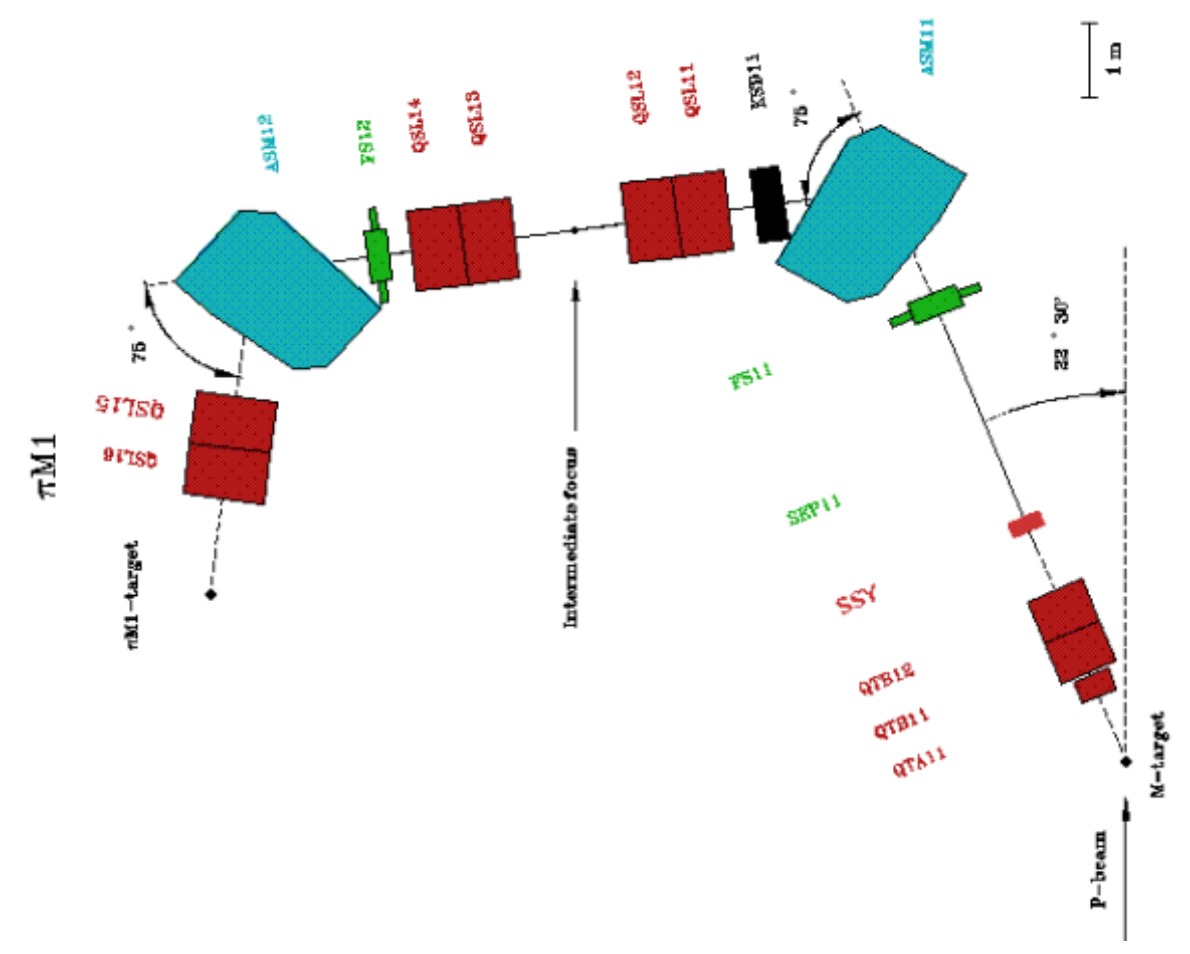

Figure 1. Layout of the beam-optics for the piM1 beam 0 line. 
dropping strongly towards lower energies as well as on the easier accessibility for the users conducting short duration experiments. The full beam optics of piM1 consist of two bending magnets ASM (75 degree each), one quadrupole triplet QTA and QTB1/2 and three systems of quadrupole doublets: QSL1/2, QSL3/4 and QSL5/6. The beam-line also contains two systems of slits FS1 and FS2 used to reduce beam intensity and improve its optics. One is placed in front of the first bending magnet and the other is positioned in the air-gap located in front of the second bending magnet—see figure below for details.

This air-gap is also used for placement of an additional energy degrader needed to differentiate momenta of various incoming particles as a result of their specific energy loss. Such degrader can be for example used for momentum separation between positive pions and protons resulting in different deflection of them by the following bending magnet. Magnet settings allow for selection of particle momenta up to $500 \mathrm{MeV} / \mathrm{c}$ while the momentum resolution of the whole system can be as good as $0.1 \%$. The charge of transmitted particles can be chosen by direction of magnetic field in the bending magnets. The focal point of the beam is located in the air about $150 \mathrm{~cm}$ behind the exit window of the last quadrupole magnet. One can reach typical beam widths of $4 \mathrm{~cm}$ to $12 \mathrm{~cm}$ of FHMS. The total length of the beam-line from the production target to the focal point is equal to about $24 \mathrm{~m}$. Beam optics settings are loaded into the system automatically. For pre-defined setup any change of the particle momentum can be completed within a very short time. PiM1 area is equipped with various cable connections (BNC, HV, network, etc.) to the measurement barrack located about $10 \mathrm{~m}$ away. Users can also relay on standard equipment such as high voltage power supplies, XY-table and beam scanners provided by the beam-line scientist crew.

\section{Experimental Setup}

Experimental setup in the piM1 area was tailored for measurement of the electrons. It consisted of the parallel plate ionization chamber located directly behind the beam exit window and the $\Delta \mathrm{E}-\mathrm{E}$ plastic scintillator telescope placed at the beam focal point. Dimensions of the $\Delta \mathrm{E}$ thin plastic were $30 \times 30 \times 2 \mathrm{~mm}^{3}$ while the cylindrically shape E-detector had thickness equal to $30 \mathrm{~mm}$ and $28 \mathrm{~mm}$ diameter. The space between both detectors was less than $5 \mathrm{~mm}$. Additional $\mathrm{NaI}(\mathrm{Tl})$ scintillation detector with the crystal size of one inch diameter and one inch thickness was used for gamma-ray detection. For measurements of the beam profile the telescope was replaced by small ( $5 \mathrm{~mm}$ diameter) plastic scintillator mounted on the XY-table. The full setup is shown in Figure 2.

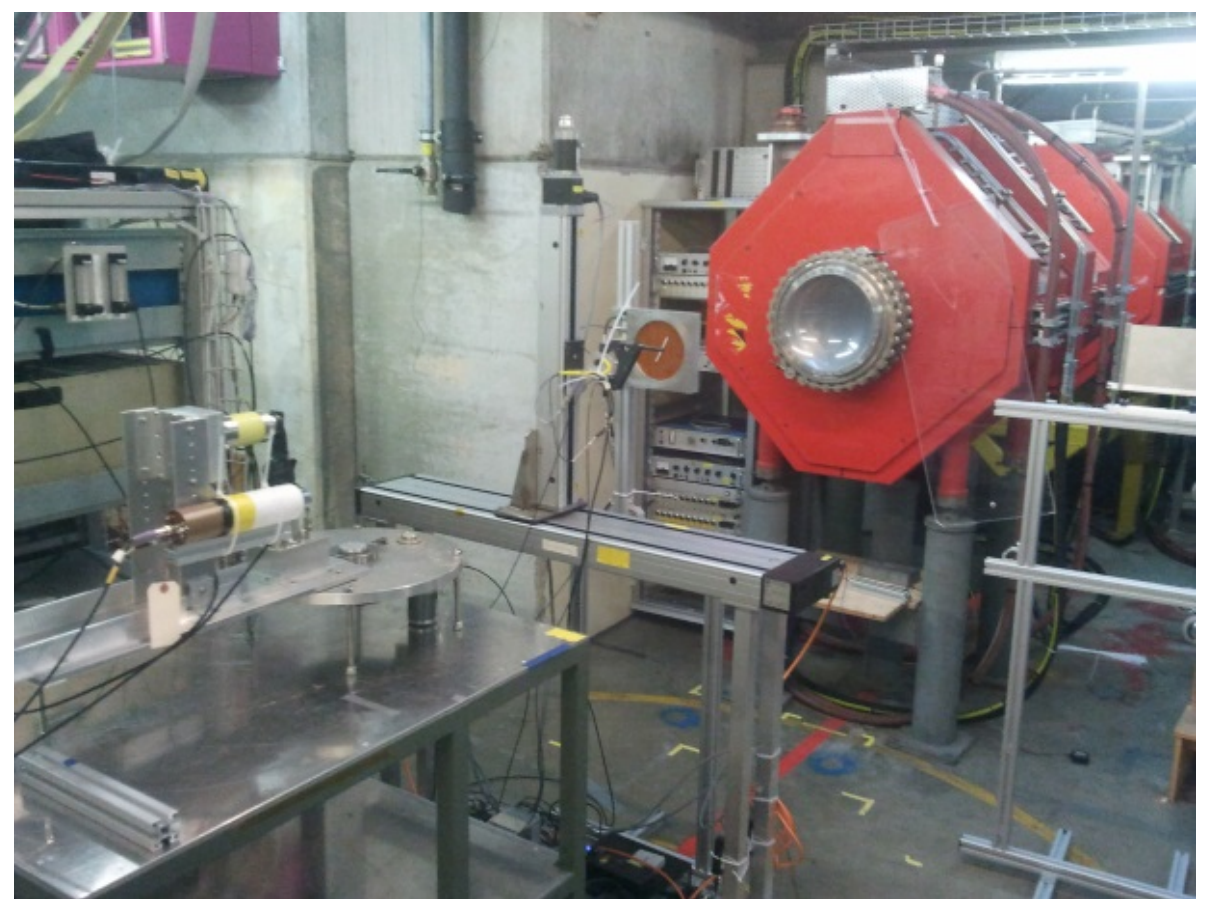

Figure 2. Experimental arrangement in the piM1 area. 
Detector system was connected with the readout electronics located in the measurement barrack placed several meters away from the area. For the ionization chamber the readout consisted of the high precision pico-amperometer with the current to voltage converter and current to frequency converter followed by the counter. Plastic telescope was read out using linear (analogue) fan-in followed by amplitude discriminators, logical coincidence unit and counters on one side and a high speed oscilloscope for the signal wave detection and analysis on the other. This instrument also allowed for analysis of the detected pulses and building of histograms from either signal amplitudes or time differences between two signals. Such data could also be stored on the disc for further analysis. The NaI (Tl) detector was read out by a system of preamplifier, amplifier and the multi-channel analyzer. Small scintillator on the XY-scanning table was connected to the discriminator and counter. It was also coupled with the XY-table control system enabling for automated measurements of the beam profiles. Beam normalization was done using additional information provided by the cyclotron control and monitoring system. All high voltage power supplies for above detectors were located directly in the piM1 area with a remote control by the experimentalists from the barrack. Initially all detectors were calibrated with radioactive sources.

Experimental arrangement for the users also allowed for easy settings of their apparatus and devices. Experimentalists had access to the tables and stages as well as additional shielding blocks made of either copper or other materials. Results from the first user tests are presented elsewhere [2].

\section{Measurement Results}

\subsection{Beam Profiles}

Beam profiles were measured with the small plastic scintillators of $5 \mathrm{~mm}$ diameter fixed on the XY-table. The system was located at the beam focal point. Vertical and horizontal scans were performed for eight momentum values in the range from $17 \mathrm{MeV} / \mathrm{c}$ to $345 \mathrm{MeV} / \mathrm{c}$. Two examples for momenta of $57 \mathrm{MeV} / \mathrm{c}$ and $115 \mathrm{MeV} / \mathrm{c}$ are shown in Figure 3. All profiles could be well described using Gauss-function shape. Fitted values of FWHMs are presented in Table 1. One can see that the profiles become wider at lower momenta. In the relevant range from $115 \mathrm{MeV} / \mathrm{c}$ to $17 \mathrm{MeV} / \mathrm{c}$ the FWHM values increase from $4.4 \mathrm{~cm}$ at the highest up to almost $12 \mathrm{~cm}$ at the lowest momentum. Such enlargement can be explained by higher probability of electron scattering at lower energies, either in air or at the entrance and exit windows of the beam optics system. It was also observed that the position of the horizontal beam center is relatively momentum independent and remains constant within about $2 \mathrm{~mm}$. The center in the vertical direction may change however by up to about $1.5 \mathrm{~cm}$. Further tests and beam optics studies are planned to explain this behavior.

Table 1. Relative contribution of electrons, muons and pions to the piM1 particle beam.

\begin{tabular}{cccccc}
\hline Momentum MeV/c & Electrons TOF & Pions TOF & Muons TOF & Electrons en. loss & Pions en. loss \\
\hline 17.25 & 100.0 & 0.0 & 0.0 & 100.0 & 0.0 \\
23.00 & 100.0 & 0.0 & 0.0 & 100.0 & 0.0 \\
34.50 & 100.0 & 0.0 & 0.0 & 100.0 & 0.0 \\
57.50 & 100.0 & 0.0 & 0.0 & 100.0 & 0.0 \\
86.25 & 100.0 & 0.0 & 0.0 & 100.0 & 0.0 \\
115.00 & 97.5 & 1.9 & 0.7 & 100.0 & 0.0 \\
143.75 & 83.8 & 14.2 & 2.1 & 82.8 & 17.2 \\
172.50 & 63.2 & 34.5 & 2.4 & 64.0 & 36.0 \\
201.25 & 46.4 & 51.4 & 2.2 & 44.2 & 55.8 \\
230.00 & 30.4 & 69.6 & 0.0 & 22.8 & 77.2 \\
\hline
\end{tabular}



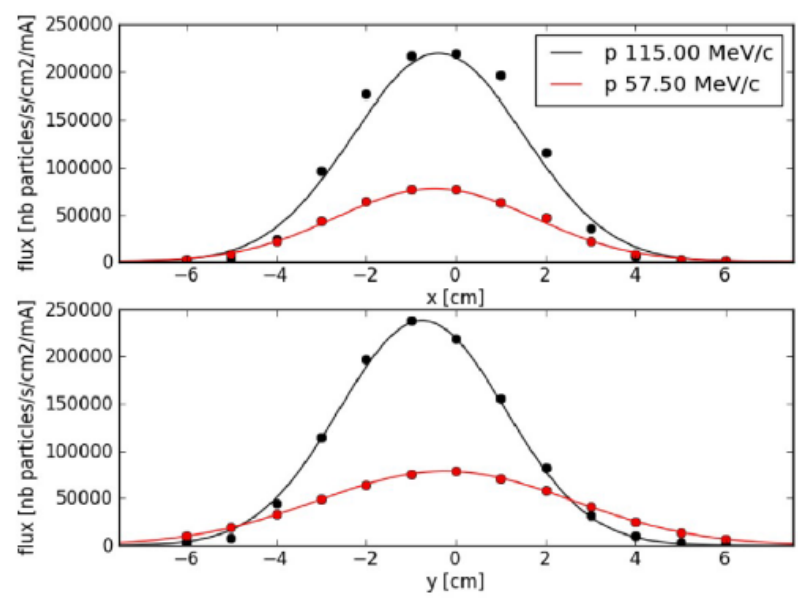

Figure 3. Example of electron beam profiles measured for two momentum values.

\subsection{Pion and Muon Fraction of the Beam}

Two methods were used to determine which types of particles composed the beam at each momentum. In the time of flight method (TOF) the flight time of particles was measured with respect to the accelerator radio-frequency signal. The start signal was provided by the small scanning detector placed during measurements at the distance of $80 \mathrm{~cm}$ upstream from the focal point or roughly $23 \mathrm{~m}$ from the production target. The stop signal was coming from the $50 \mathrm{MHz}$ frequency of the synchro-cyclotron. This signal caused 20 nsec long periodicities in measured TOF histograms. An example of two TOF spectra for momenta equal to $172 \mathrm{MeV} / \mathrm{c}$ and $230 \mathrm{MeV} / \mathrm{c}$ is presented in Figure 4.

For each momentum three well distinguished peaks can be observed. As for all used momenta the electrons travel with almost the speed of light their position in the spectrum remains unchanged-see peaks centered around 0 ns value in figure. The speed of pions and muons is strongly momentum dependent. It is also well seen that positions of their peaks are shifted. Relative distance to the electron peak can be verified in calculations using particle momentum, its mass and the trajectory length. This allows for assigning of the peak either to pions or muons as well as, from integrated peak intensities, determination of relative contribution of specific particle to the total beam intensity-see Table 1 for results. As the peaks are not only well separated but the space inbetween is free of background events, the gamma-ray contamination of the beam is relatively low.

In the second method, the particles were identified using their energy loss in the thick plastic scintillator of the $\Delta \mathrm{E}$-E telescope. An example of the spectrum measured at $172 \mathrm{MeV} / \mathrm{c}$ is shown in Figure 5. Although one can see two peaks, their separation is not as good as in the time of flight method mainly due to the insufficient detector thickness. To separate the peaks and find their relative contributions one performed fits of the Vavilov function-see the red curve in figure. In the first step the fits were done to the spectra of pure electrons i.e. for momenta smaller than $100 \mathrm{MeV} / \mathrm{c}$. After normalization of this function to the electron peak maximum for the spectra with double peaks, the contribution of other particles was found by subtraction. Due to limits in energy resolution, this method was not able to provide any information on the muon presence in the beam especially as their fraction was of the order of a few percent at the maximum.

Nonetheless, using above method of analysis gives the results that agree with measurements with the time of flight method on the level of less than $10 \%$. This discrepancy is comparable to experimental errors depending on statistical accuracy, energy resolution and fit quality. However, the energy loss measurement is considered only as a supporting measurement and for further use the TOF data should be taken.

Relative beam contributions from different particles are shown in Figure 6 in which the results from both separation methods are presented. One can see that electron spectra with momenta up to $100 \mathrm{MeV}$ are virtually free of any pion and muon contamination. Above results also agree with previous measurements done for the MUSE experiment [3] with respect to the electron fraction in the beam. Some differences are seen for the relative distribution between pions and muons at higher energies. Although irrelevant for electron beam this issue is subjected to further investigation. 
Time of flight spectra

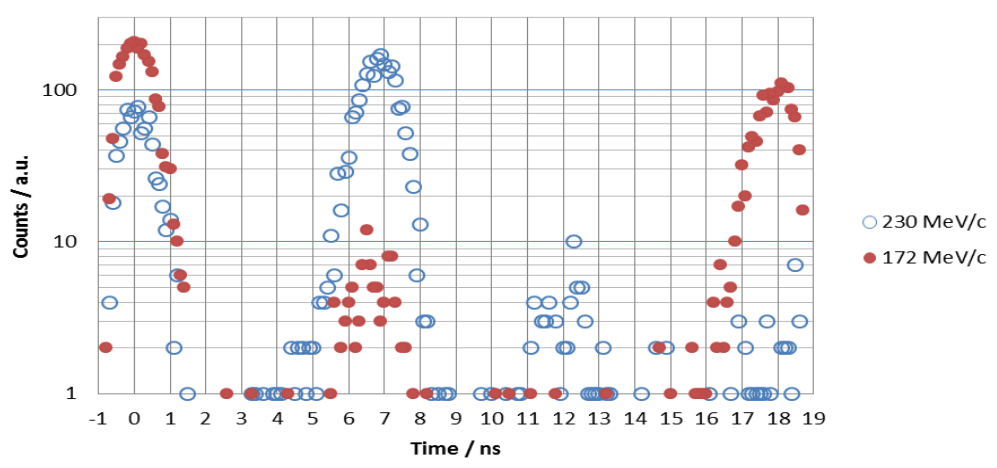

Figure 4. Time of flight spectra measured at two different momenta.

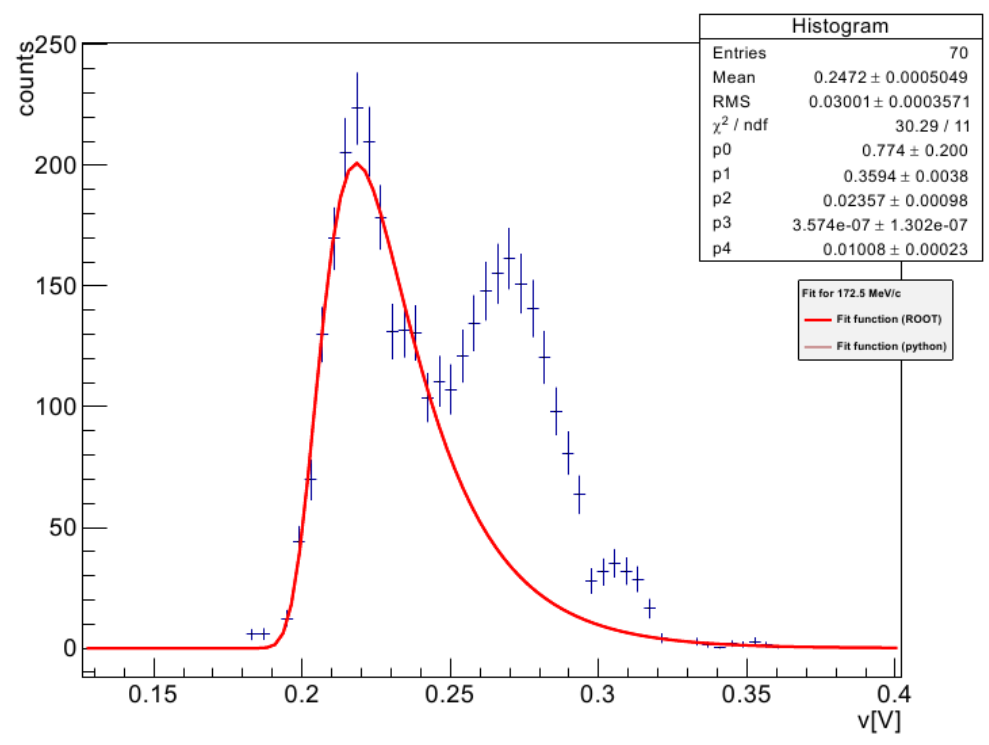

Figure 5. Spectrum of energy loss measured by the thick detector of the telescope at momentum $172 \mathrm{MeV} / \mathrm{c}$.

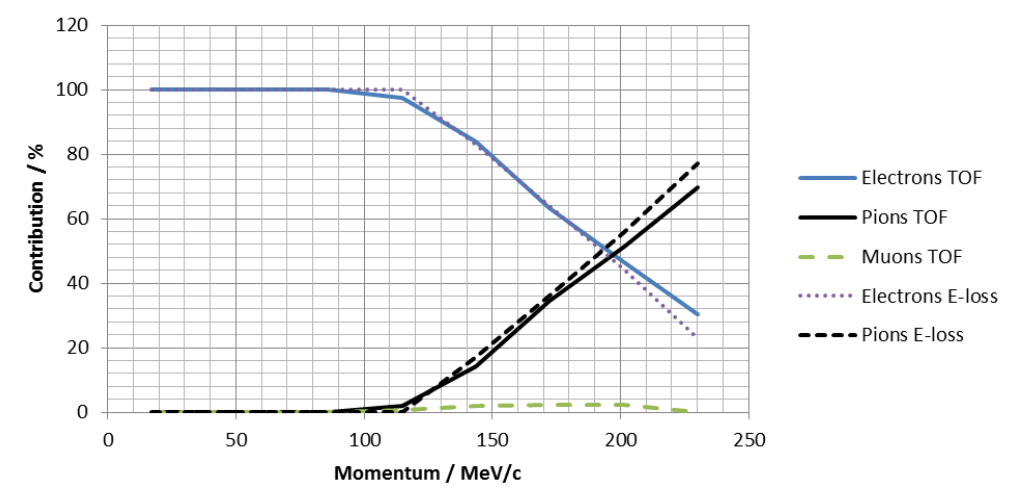

Figure 6. Relative contributions of electrons, muons and pions to the piM1 particle beams.

It is also possible to reduce the non-electron fractions in the beam for momenta higher than $100 \mathrm{MeV} / \mathrm{c}$. The most efficient way is using of the electrostatic separator deflecting particles depending on their mass. Such devices are at the moment installed in other secondary beam areas of PSI. For experiments requiring higher mo- 
mentum pure electron beams such area may be accessible upon approved user proposal and beam request. Another method of removal is based on utilization of the energy degrader in the beam. It reduces and differentiates momenta of passing particles. By proper tuning of the deflecting magnet placed further downstream, desired particle type can be selected while the others will be removed from the beam-line. This method was successfully tested using positively charged beams and separating protons from positive pions at momentum values of $350 \mathrm{MeV} / \mathrm{c}$.

\subsection{Electron Fluxes}

Electron fluxes and intensities were measured using small plastic scintillator on the XY-table. Measurements were performed at the focal point of the beam. The main proton beam current was also measured in parallel for normalization purposes. The full intensity of the secondary beams in the area was measured using the same method and by applying beam scans. The intensity value was obtained by integration of the scanned profiles over vertical and horizontal directions. Both results are presented in Figure 7. They are given for the maximum proton beam current of $2.1 \mathrm{~mA}$. Data presented in Table 2 are given for the normalized $1 \mathrm{~mA}$ value of the proton current. As one can see the electron intensity rises with the momentum and becomes relatively constant at the value of about $75 \mathrm{MeV} / \mathrm{c}$. The increase is relatively smooth-one order of magnitude from $25 \mathrm{MeV} / \mathrm{c}$ to 75 $\mathrm{MeV} / \mathrm{c}$. The electron flux shows different behavior and is characterized by much faster changes with momentum. It is caused by additional enlargement of the beam profiles and increased scattering in air towards lower momentum. It gives the maximum flux at $25 \mathrm{MeV} / \mathrm{c}$ to be only about $10^{4} / \mathrm{cm}^{2} / \mathrm{s}$.

Having the piM1 beam-line fully contained in the vacuum would largely reduce electron scattering as two thick windows and air-gap of about $2 \mathrm{~m}$ in length would be removed. It would result in higher fluxes and smaller profiles at low energies. Such the modification of the beam-line is however not foreseen for the near future. Therefore lower energy beams are mostly useable for tests of shielding penetration and detector calibration but not suitable for total dose studies at high dose rate values.

As some experiments need to work with even lower intensities one can provide them by reducing the opening of the beam line slits. This way the electron flux can be reduced to very low values. The total beam intensity can

Table 2. Electron beam parameters in piM1 area.

\begin{tabular}{ccccc}
\hline Momentum MeV/c & Intensity s/mA & Flux cm²/s/mA & FWHMx cm & FWHMy cm \\
\hline 17.3 & $1.16 \mathrm{E}+05$ & $7.21 \mathrm{E}+02$ & 10.4 & 13.2 \\
23.0 & $3.28 \mathrm{E}+05$ & $2.57 \mathrm{E}+03$ & 9.0 & 12.9 \\
34.5 & $1.16 \mathrm{E}+06$ & $1.56 \mathrm{E}+04$ & 6.6 & 9.6 \\
57.5 & $3.08 \mathrm{E}+06$ & $7.88 \mathrm{E}+04$ & 5.2 & 6.6 \\
86.3 & $5.13 \mathrm{E}+06$ & $1.69 \mathrm{E}+05$ & 4.2 & 5.1 \\
115.0 & $5.18 \mathrm{E}+06$ & $2.42 \mathrm{E}+05$ & 4.4 & 4.3 \\
\hline
\end{tabular}
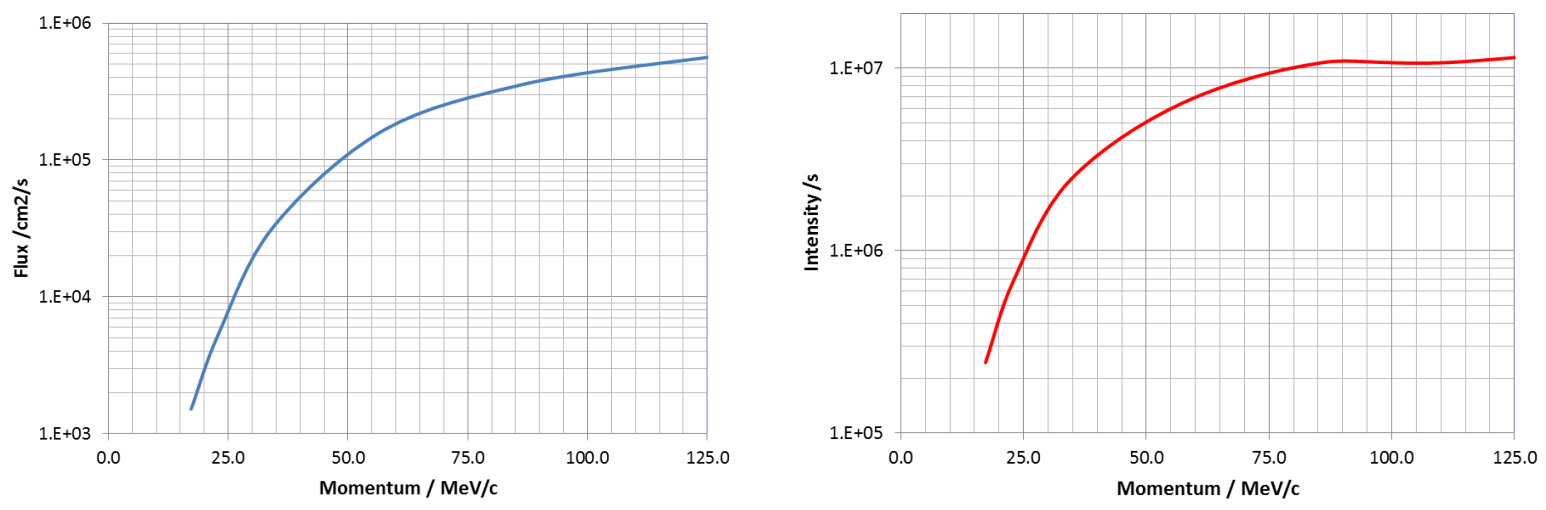

Figure 7. Maximum values of the electron flux and intensity for proton beam intensity of $2.1 \mathrm{~mA}$. 
be monitored with the ionization chamber usually placed at the exit of the bam from the last quadrupole. It is however necessary to perform flux and profiles measurement anew as the beam optics is changed with each modification of the slit openings.

\section{Summary and Conclusions}

Secondary beam area piM1 in the Experimental Hall at thePaul Scherrer Institut was selected to test and characterize electron beams of high momenta. The goal was its possible use for components exposures and instrument testing for space applications. It was found that with existing beam optics the electron beams are free of other particle contamination on the level of much less than $1 \%$ for momentum values up to about $115 \mathrm{MeV} / \mathrm{c}$. Relative contamination of the beam by secondary gamma-rays was also found to be on a very small level. Therefore the area fulfills the criteria for providing electrons within energy range suitable for e.g. missions flying to Jupiter such as JUICE. The maximum beam fluxes vary from about $10^{3} / \mathrm{cm}^{2} / \mathrm{s}$ at momentum value of $17.5 \mathrm{MeV} / \mathrm{c}$ up to $4 \times 10^{5} / \mathrm{cm}^{2} / \mathrm{s}$ at $155 \mathrm{MeV} / \mathrm{c}$. Beam profile FWHM also shows momentum dependence changing from about 12 $\mathrm{cm}$ at the lowest to about $4 \mathrm{~cm}$ at the highest momentum values.

The piM1 area provides various equipment and utilities for the users such as cable connections, basic power supplies and electronics infrastructure as well as movable stages and numerous shielding arrangements. Further studies of beam parameters are ongoing, including characterization of the positively charged beams and determination of the beam proton fraction. In addition, measurements of gamma-ray and electron spectra behind predefined shielding as well as verification of the collimator parameters at various initial electron energies are being performed.

The area is planned to be used for further space experiments aimed for space mission related studies such as shielding optimization, performance characterization of instruments in presence of high energy electron background as well as calibration of detection systems.

\section{References}

[1] Deiters, K. (2013) piM1 Beamline. http://aea.web.psi.ch/beam2lines/beam pim1.html

[2] Tulej, M., et al. (2014) August 2014 submitted to nuclear instrument and methods.

[3] M. Kohl for MUSE Collaboration (2014) Muon elastic scattering with MUSE at PSI. EPJ Web of Conferences 66, 06010. 